\title{
FLUJO DE FONDOS: PODEROSA HERRAMIENTA DE GESTIÓN EN LOS NEGOCIOS (PARTE II)
}

\author{
José Ángel Porlles Loarte (*)
}

E-mail: joseporlles@yahoo.com

\section{RESUMEN}

En la primera parte de esta Serie Flujo de Fondos se incidió en el marco conceptual de los diferentes enfoques de construcción del Estado de Movimiento de Fondos. En esta segunda parte se procede al análisis del impacto y la conexión de las diversas cuentas del Balance General y del Estado de Resultados (EPG) en el Estado de Movimiento de Fondos, para su correcta interpretación y entendimiento de los cambios, obteniendo así información vital que contribuye a un mejor análisis financiero de lo ocurrido con el origen y usos de fondos, así como su impacto en la estructura financiera de la empresa. El manejo sistemático y reflexivo de esta herramienta de análisis potenciará a los profesionales vinculados en la toma de decisiones gerenciales.

Palabras clave: Fondos generados por operaciones, caja generada por operaciones, liquidez.

\section{ABSTRACT}

In the first part of this Funds Flow Series, was impacted in the conceptual framework of the different focus of construction of the Funds Movement State. In this second part al proceeds analysis of the impact and the connection of the diverse accounts of the General Balance and of the State of Results (EPG) in the Funds Movement State, for its correct interpretation and understanding of the changes, obtaining thus vital information that contributes to a better financial analysis of what happened with the origin and uses of funds, as well as its impact in the financial structure of the company. The reflexive and systematic management of this tool of analysis will harness the professionals linked in it takes of managerial decisions.

Key words: Funds generated by operations, cash generated by operations, liquidity.

* Magíster en Administración. Profesor adscrito al Departamento de Análisis y Diseño de Procesos en la UNMSM. Consultor e Investigador en temas empresariales (evaluación de inversiones y negocios). 
La dura competencia de las empresas en las industrias tiende a que éstas consoliden cuadros profesionales con un manejo adecuado de herramientas de análisis en la resolución de problemas. En el área de las finanzas se requiere diferenciar entre el análisis contable, el análisis económico y el análisis financiero. En el presente documento se resaltan los argumentos para una cabal formulación del análisis financiero en el movimiento de fondos, sobre la base de los estados financieros. Se deja constancia que no se pretende agotar los diferentes ángulos para incrementar la certidumbre en los resultados que conducen a una razonable toma de decisiones financieras, tomando en cuenta la variedad de situaciones en que se pueden encontrar las empresas en el mundo real.

Con el propósito de ampliar su cobertura de uso para los casos más frecuentes, se toma como base la situación de una empresa manufacturera, para lo cual se utiliza la información base que se consignó en la primera parte del presente trabajo ${ }^{1}$, que también se incluye en el presente documento como Anexo 1.

\section{FONDOS GENERADOS POR OPERACIONES (FGO)}

Con mucha frecuencia se dice que la diferencia entre el flujo de ingresos de efectivo menos el flujo de egresos en efectivo proporciona un saldo denominado de diferentes modos: saldo de caja, cash, saldo de efectivo o fondos generados; y los cuadros de flujo toman el nombre respectivamente de flujo de ingresos y egresos, flujo de efectivo, cash flow o flujo de fondos generados por operaciones (FGO) o simplemente flujo de fondos.

Aparentemente no hay diferencias pero en realidad sí las hay. Entonces es apropiado usar y entender adecuadamente dicha terminología para conocer con certeza de qué se está hablando, para lo cual primero deben precisarse ciertos conceptos.

El Balance muestra la situación de los fondos a una cierta fecha y estos son valores de stock, no de flujo, por lo tanto no constituye un estado de flujo.

El estado de flujo grafica los cambios que tuvieron lugar en la partidas del Balance entre dos fechas determinadas.
El Estado de Resultados (EPG) es un estado del flujo que explica los cambios que se ha producido en la cuenta de utilidades no distribuidas en un cierto periodo de tiempo, dado por los ingresos por ventas como aumentos y por los gastos como reducciones.

El Estado de Flujos de Efectivo como cuadro de ingresos y egresos de caja puede determinarse sumando todos los débitos y créditos de la partida de caja, es decir ingresos en efectivo y egresos o desembolsos en efectivo de la empresa en un cierto periodo de tiempo, produciendo un saldo de caja que representa el cambio de efectivo en dicho periodo, lo que se traduce como variación en la cuenta Caja/Bancos.

El Estado Flujos de Efectivo como se ha conceptuado en la Parte I del presente trabajo ${ }^{2}$ difiere del estado de ingresos y egresos anotado en el párrafo anterior, no en su cuantía, pero sí en su concepto, en cuanto que su construcción es a partir de determinadas cuentas del Balance y del Estado de Resultados y no de asientos vinculadas a la cuenta caja, explicando además de manera relevante las causas de la variación de dicho saldo de caja, proporcionando información que ayuda a un mejor análisis financiero y razonable interpretación de lo sucedido con el origen y aplicación de los fondos obtenidos. Definitivamente todas las partidas de gastos excepto la depreciación y amortizaciones de intangibles impactan la cuenta caja o las demás cuentas del circulante, por tanto afectan el movimiento del capital circulante.

\section{¿Cómo se obtienen los FGO?}

Un mecanismo muy utilizado por su practicidad es el que se comenta a renglón seguido. Se conoce que si a la utilidad del ejercicio se suma la depreciación de activos fijos y amortizaciones de intangibles, se obtienen los FGO. Esta situación se ilustra en el Cuadro 1 (tomando las cifras del Anexo I para el periodo 2003).

Cuadro 1. Cálculo del FGO.

\begin{tabular}{lr}
\hline Utilidad del ejercicio (Utilidad Neta) en \$ & 110,500 \\
Más Depreciación y amortizaciones & 50,00 \\
Fondos Generados por Operaciones (FGO) & 160,500 \\
\hline
\end{tabular}


En realidad los Fondos Generados por Operaciones (FGO) es el resultado de determinar los ingresos como fuente de fondos, restando los gastos operativos que emplearon fondos (costo de producción, costos operativos e impuestos).

El mecanismo utilizado en la determinación de los FGO con frecuencia confunde acerca de la naturaleza de la depreciación, de ahí que Anthony y Reece ${ }^{3}$ hacen un deslinde sobre dicho cálculo. Anotan que sumar la depreciación a la utilidad neta es solo una operación aritmética, ya que es equivalente a restar la depreciación de la cuenta de gastos en el Estado de Resultados, reduciendo los gastos y por tanto aumentando la utilidad. Añaden además que en la confección del Diario para registrar el gasto por depreciación, no se afecta la cuenta de activo corriente ni la cuenta de pasivo corriente; es decir, la depreciación no resulta ser una fuente de capital de trabajo ni uso de ese capital. Por consiguiente, la depreciación no constituye entonces una fuente o empleo de fondos.

\section{¿Cuál es la interpretación del Fondo de Maniobra (FM)?}

El Fondo de Maniobra (FM) o Capital de Trabajo (CT) suele conceptuarse de las formas siguientes:

$\begin{array}{ll}\text { CT = AC-PC } \quad \text { donde: } & \text { AC: activo corriente } \\ \text { FM = RP-AI } & \text { PC: pasivo corriente } \\ & \text { RP: recursos } \\ & \text { permanentes } \\ & \text { AI: activos inmovili- } \\ & \text { zados (fijos e } \\ & \text { intangibles) }\end{array}$

Bajo la definición (AC-PC) el FM se identifica con inversión de recursos circulantes, aquellos que no son financiados con fondos obtenidos dentro de la actividad propia del negocio dentro de la estructura patrimonial circulante ${ }^{4}$, como se señala en el Cuadro 2.

Cuadro 2. Estructura patrimonial circulante

\begin{tabular}{lrrc}
\hline \multicolumn{1}{c}{ Concepto en \$ } & \multicolumn{1}{c}{ 2002 } & 2003 & Variación \\
\hline Activo Circulante (AC) & 370,000 & 450,000 & 80,000 \\
Pasivo Circulante (PC) & 150,000 & 210,000 & 60,000 \\
Fondo de Maniobra & 220,000 & 240,000 & 20,000 \\
CAJA/ BANCOS & 30,000 & 40,000 & 10,000 \\
\hline
\end{tabular}

Cuantitativamente deben obtenerse los mismos resultados si se considera el concepto relevante de los recursos permanentes e inmovilizados. En el esquema de la definición (RP-AI), el FM se identifica como una fuente fi-

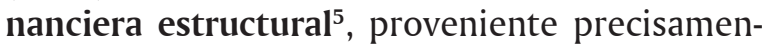
te de parte de los recursos permanentes que forma parte de la estructura patrimonial permanente (Cuadro 3).

Cuadro 3. Estructura patrimonial permanente.

\begin{tabular}{lrrr}
\hline Concepto en \$ & \multicolumn{1}{c}{2002} & \multicolumn{1}{c}{2003} & Variación \\
\hline Recursos Permanentes (RP) & 720,000 & 790,000 & 70,000 \\
Pasivo a largo plazo (PLP) & 70,000 & 90,000 & \\
Capital social & 400,000 & 400,000 & \\
Utilidades retenidas & 250,000 & 300,000 & \\
& & & \\
Inmovilizados (AI) & 500,000 & 550,000 & 50,000 \\
Inversiones permanentes & 50,000 & 50,000 & \\
Edificaciones \&Planta Neto & 450,000 & 500,000 & \\
Fondo de Maniobra (FM) & 220,000 & 240,000 & 20,000 \\
\hline
\end{tabular}

Este concepto más relevante $(\mathrm{FM}=\mathrm{RP}-\mathrm{AI})$ pone énfasis en que el Fondo de Maniobra es aquella porción de los recursos permanentes que financia el activo circulante de la empresa.

Arbulú y Stok ${ }^{6}$ anotan “... que esta segunda definición aclara mucho más la verdadera naturaleza del CT”, aclarando además que es un concepto de pasivo más que un concepto de activo, correspondiendo su responsabilidad a la dirección general y directivos financieros. En cambio, agregan, la gestión de las inversiones y recursos relacionados con las operaciones (cuestiones de funcionamiento u operativas para el caso $\mathrm{CT}=\mathrm{AC}-\mathrm{PC}$ ) son responsabilidad de todos los directores de la empresa.

Siguiendo con la ilustración, un aumento del FM significa aplicación de fondos (consumo de recursos). Si hubiese disminuido, se presenta una fuente $\mathrm{u}$ origen de fondos, dado que se estaría tomando la actual liquidez para financiar otras actividades, en tanto la cuenta Caja/Bancos del Balance indica también un aumento de $\$ 10,000$. No debe perderse de vista que la idea central de todo empresario y analista es conocer de qué forma el desarrollo de las decisiones de inversión/financiamiento han incidido en la situación de equilibrio de la empresa, de manera que la misma esté en condiciones de hacer frente a sus obligaciones sin poner en riesgo su futuro operativo. 
Entonces la clave en el análisis radica en conocer no solo los cambios de las partidas que se han producido en las cuentas patrimoniales durante un periodo de tiempo, sino en conocer e identificar cómo los cambios en las cuentas patrimoniales han modificado la estructura patrimonial circulante y permanente. Así pues, se necesita detectar cuáles han sido las fuentes financieras obtenidas durante el ejercicio de una gestión, así como las partidas en las que se han invertido dichos recursos.

Los flujos relacionados con transacciones que afectan la estructura patrimonial permanente de la empresa, proporcionan o utilizan fondos que son los que en definitiva determinan la situación actual del FM, como capital que debe garantizar su capacidad de pago, cuyo manejo improcedente puede poner en peligro la estabilidad financiera de la empresa.

Este razonamiento sustenta la necesidad de elaborar un cuadro explicativo de las causas de variación del FM que informará de las variaciones de las partidas de la estructura circulante de la empresa, en tanto que su justificación estará tanto en los cambios en la estructura patrimonial permanente como en los resultados de gestión en la propia actividad del negocio.

\section{¿Dónde impactan entonces los FGO: en el FM o en la cuenta Caja / Bancos?}

El saldo de caja, cash o FGO antes indicado, pareciera configurar que la cuenta Caja/ Bancos ha sufrido un aumento de $\$ 160,500$ como se señala en el Cuadro 1. No obstante, el análisis de dicha cuenta consigna solo una variación incremental de $\$ 10,000$, lo que se aprecia en el Cuadro 2. Por tanto, los FGO no van directamente a la cuenta Caja/ Bancos. La pregunta inmediata es entonces ise dirigió al FM?. Sin embargo, la cifra de los FGO no coincide con la variación del FM, por tanto, ¿cuál sería la interpretación?

Analizando los componentes de los FGO, las partidas de ingresos y gastos que lo determina no necesariamente pueden haberse traducido totalmente en caja, pero definitivamente registra una interrelación con las cuentas del circulante y su incidencia dependerá de las políticas de crédito a las ventas, políticas de inventario y políticas de pago a proveedores. En consecuencia, el efecto neto que se deriva es una variación en el FM.
Enfatizando, el destino de los Fondos Generados por Operaciones (FGO) es el FM, por las razones siguientes:

1. Los FGO o saldo de efectivo son el resultado de la diferencia entre los ingresos y los gastos que ha supuesto desembolso durante el periodo o que se va a producir dicho desembolso en el corto plazo.

2. Las ventas del periodo pueden no haberse cobrado en su totalidad.

3. Parte de las compras del periodo es posible que no se hayan pagado en su totalidad a los proveedores.

4. Es posible haber recibido adelantos de pago por ventas, cuyos productos (existencias) se encuentran todavía en almacén.

5. De haber un aumento en el crédito puede no haberse cancelado el íntegro de las compras de activos fijos o existencias.

6. Puede haberse vendido parte de los activos fijos.

7. El movimiento de tales partidas de ingresos y gastos que determinaron los FGO no necesariamente se refleja en la partida Caja/ Bancos.

8. No obstante, las partidas ingresos y gastos necesariamente se traducen en el cambio de alguna partida del activo o del pasivo circulante.

Es importante precisar que los FGO son equivalentes a la variación del FM, sólo cuando sucede lo siguiente:

- No hay movimiento en la aportación de capital social ni obligaciones a largo plazo.

- No hay variación en los activos inmovilizados.

- No hay reparto de dividendos.

- Sólo las operaciones ordinarias (operación comercial) son la única fuente y consumo de recursos durante el periodo.

\section{CAJA GENERADA POR OPERACIONES (CGO)}

Un concepto es los FGO, otra la CGO y otra la cuenta Caja /Bancos. Para un mejor entendimiento de lo anotado es necesario precisar previamente algunos conceptos complementarios. Es conveniente dejar establecido que la estructura del ciclo de operaciones es determinante en el nivel requerido de fondos como FM, en función de cómo se manejen las actividades de compra, fabricación y venta que en conjunto 
-desde un punto de vista financiero- determinan un ciclo de caja que no necesariamente es equivalente al ciclo de operaciones ${ }^{7}$. Las políticas financieras deben definir las condiciones de los niveles de crédito, inventario y pago a proveedores.

Es también importante entender que aproximadamente conforme aumenta o disminuye el volumen de ventas, varía proporcionalmente el nivel de las cuentas de crédito por ventas, existencias y crédito de proveedores. En este punto se introduce el concepto de la Necesidad Operativa de Fondos (NOF), que es componente fundamental del FM. La NOF en este trabajo, exceptuando la partida Caja/Bancos, está determinada de la manera siguiente: por las cuentas más significativas del circulante (clientes, existencias y proveedores, cuyos movimientos y stock varían proporcionalmente en función de las ventas), y por las demás cuentas menores del circulante agrupadas en el presente documento con la denominación Otros APC (su desarrollo se realiza más adelante). Arbulú y $\mathrm{Stok}^{8}$ proponen un modelo de cálculo de la NOF y el Capital de Trabajo, definiendo la NOF según el enfoque que sigue:

“... es la diferencia entre activos corrientes y pasivos corrientes derivados de las operaciones de la empresa, sin tener en cuenta para nada las decisiones de financiación o de colocación de excedentes de caja, aunque se trate de decisiones de corto plazo".

Si bien en dicho modelo se plantea una mayor rigurosidad para el cálculo de la NOF, para fines prácticos, la propuesta aquí planteada no pierde eficacia frente al objetivo propuesto.

Entonces las necesidades de fondos para financiar las operaciones propias del negocio estarán en relación proporcional al nivel de ventas $^{9}$. Obviamente que un crecimiento sostenido de las ventas requiere financiamiento de tipo estructural. En consecuencia, en este nivel del análisis se está en condiciones de mencionar que la CGO representa fondos expresados como liquidez, resultado de aplicar al nivel de los FGO la variación de las NOF que corresponde a los cambios de las partidas del circulante (excepto la cuenta Caja/Bancos).

\section{¿La variación en la cuenta Caja/Bancos es a partir de la CGO?}

En la determinación de la variación de la partida Caja/Bancos a partir de la CGO se deben adicionar otras fuentes de fondos derivados por aumento de capital, créditos a largo plazo y ventas de activos permanentes para tener un total de caja; para luego restarle las aplicaciones de fondos tales como pago de dividendos, compras de activos e inversiones permanentes.

\section{¿Son equivalentes los FGO a la CGO?}

Los FGO son equivalentes a la CGO, sólo cuando sucede lo siguiente:

- Cuando no hay movimiento en las partidas de activos inmovilizados y recursos permanentes (pasivo a largo plazo y patrimonio) y distribución de dividendos.

- Cuando en las partidas de ingresos y gastos las transacciones son al contado, es decir, no hay variación en las cuentas circulante que se agrupan bajo la denominación Otros APC.

ANÁLISIS DE LA VARIACIÓN DE LAS CUENTAS DEL EPG Y DEL CIRCULANTE EN EL BALANCE GENERAL

\section{¿Porqué los FGO no impactan directamente en la cuenta Caja/ Bancos?}

Para responder con énfasis la pregunta, se debe examinar el impacto de las partidas ingresos y gastos que determinaron los FGO, sobre las cuentas del circulante del Balance en términos de dinero.

\section{Análisis de las Ventas}

Es necesario responder a ¿qué ha sucedido con las ventas del período?, ¿se encuentran en efectivo? Debe entenderse que la cuenta ventas está vinculada a la cuenta clientes, por lo que debe examinarse su variación (Cuadro 4).

Seguidamente se puede calcular la caja generada por las ventas (CGV) que se visualiza en el Cuadro 5.

Cuadro 4. Cuenta clientes.

\begin{tabular}{lccc}
\hline Concepto en \$ & 2002 & 2003 & Variación \\
\hline Cuenta por cobrar (clientes) & 170,000 & 200,000 & 30,000 \\
\hline
\end{tabular}

Cuadro 5. Caja generada por las ventas

\begin{tabular}{lr}
\hline Ventas en \$ & $2,000,000$ \\
Menos Aumento de clientes & 30,000 \\
Caja Generada por Ventas (CGV) & $1,970,000$ \\
\hline
\end{tabular}




\section{Análisis del Costo de Ventas}

Es fundamental que esta partida excluya los cargos por depreciación y otras partidas que no implican desembolsos. ¿Se desembolsó los $\$ 1,500,000$ del costo de ventas? Para responder esta pregunta se deben analizar las partidas del Balance vinculadas al costo de ventas: existencias y proveedores, cuyas variaciones se presentan en el Cuadro 6. Por tanto, recién se puede computar el costo de ventas en términos de caja, aspecto que se revela en el Cuadro 7.

Cuadro 6. Variaciones en existencias y proveedores.

\begin{tabular}{lrcr}
\hline Concepto en \$ & 2002 & 2003 & Variación \\
\hline $\begin{array}{l}\text { Cuentas por pagar } \\
\text { (proveedores) }\end{array}$ & 50,000 & 80,000 & 30,000 \\
Existencias (Inventarios) & 130,000 & 180,000 & 50,000 \\
\hline
\end{tabular}

Cuadro 7. Costo de ventas en términos de caja.

\begin{tabular}{lr}
\hline Costo de ventas en $\$$ & $1,500,000$ \\
Más Aumento de Existencias & 50,000 \\
Menos Aumento de Proveedores & $(30,000)$ \\
CAJA Consumida por Costo de Ventas & $\mathbf{1 , 5 2 0 , 0 0 0}$ \\
\hline
\end{tabular}

\section{Análisis de otras cuentas del Activo y Pasivo} Circulante (Otros APC)

Pueden existir otras cuentas diferentes de la triada: clientes/ existencias/ proveedores que han incidido en el circulante del Balance, tales como gastos anticipados, gastos diferidos, sobregiros, impuestos por pagar, documentos por pagar, entre otras cuentas. El efecto neto de las variaciones de dichas cuentas se ha denominado Otros APC (Cuadro 8), tal como se ha señalado anteriormente.

Cuadro 8. Variación de Otros APC.

\begin{tabular}{lrrr}
\hline Concepto en \$ & 2002 & 2003 & Variación \\
\hline $\begin{array}{l}\text { Sub total activos APC } \\
\text { Inversiones en valores }\end{array}$ & 10,000 & 10,000 & \\
Gastos pagados por adelantado & 30,000 & 20,000 & $(10,000)$ \\
& & & \\
Sub total pasivos APC & & & 30,000 \\
Sobregiros & 20,000 & 30,000 & 10,000 \\
$\begin{array}{l}\text { Documentos por pagar } \\
\text { Gastos devengados por pagar }\end{array}$ & 40,000 & 70,000 & 30,000 \\
Efecto neto Otros APC & & 30,000 & $(10,000)$ \\
\hline
\end{tabular}

En este caso la variación neta de estas partidas es $\$ 40,000$ y su resultado se interpreta como sigue: la disminución de los gastos pagados por adelantado representa una fuente de fondos; los aumentos en sobregiros y documentos por pagar también representan fuente de fondos, en tanto la disminución de los gastos devengados por pagar configura uso de fondos.

\section{Análisis de los Gastos de Explotación}

En el Estado de Resultados (EPG), los gastos de explotación comprenden los denominados gastos de operación (gastos administrativos y gastos de ventas, gastos financieros e impuestos), excluyendo los cargos por depreciación y amortizaciones que corresponden a cuentas no desembolsables. La referida partida está vinculada a las restantes cuentas del circulante Otros APC, cuyas variaciones se han calculado en el acápite anterior. Luego, el efecto de la cuenta Otros APC en el rubro gastos de explotación en términos de caja se ilustra en el Cuadro 9.

Cuadro 9. Gastos de explotación en términos de caja.

\begin{tabular}{ll}
\hline Gastos de explotación (desembolsables) \$ & 339,500 \\
Menos Aumento Otros APC & $(40,000)$ \\
CAJA Consumida por Gastos de Explotación & 299,500 \\
\hline
\end{tabular}

\section{Calculando la Caja Generada por Operacio- nes (CGO)}

Una forma alternativa de calcular la CGO tomando el esquema del Estado de Resultados en términos de liquidez se desarrolla en el Cuadro 10.

Cuadro 10. Cálculo de la CGO.

\begin{tabular}{lr}
\hline Caja generada por las ventas en \$ & $1,970,000$ \\
Menos Caja usada por costo de ventas & $1,520,000$ \\
Menos Caja usada por gastos de explotación & 450,000 \\
CAJA Generada por Operaciones (CGO) & 150,500 \\
\hline
\end{tabular}

¿Es posible otra forma de computar la CGO?

Por supuesto que sí. La forma alternativa usual y práctica de presentar la CGO es asociando los fondos generados por las operaciones 
(FGO) con la necesidad operativa de fondos (NOF), esquema que es recomendable utilizar para apreciar en su conjunto las interrelaciones de las cuentas del circulante del Balance y su efecto en el EPG (Cuadro 11).

Cuadro 11. Forma alternativa de cálculo: CGO.

\begin{tabular}{lrr}
\hline $\begin{array}{lr}\text { Fondos Generados por Operaciones } \\
\text { (FGO) en \$ }\end{array}$ & 160,500 \\
Variación de la NOF & $(10,000)$ \\
Menos Aumento de Clientes & $(30,000)$ & \\
Menos Aumento de Existencias & $(50,000)$ & \\
Más Aumento de Proveedores & 30,000 & \\
Más Aumento Otros APC & 40,000 & \\
CAJA Generada por Operaciones (CGO) & & 150,500 \\
\hline
\end{tabular}

¿Ahora es factible explicar la variación de la cuenta Caja/Bancos a partir de la CGO?

Es factible. Se aprecia que la cuantía de la CGO es diferente que la variación de la citada cuenta Caja/Bancos por $\$ 10,000$. No hay equivalencia habida cuenta que se registran variaciones en otras partidas no circulantes como fuente y uso de fondos. Remarcando nuevamente, el resultado de la CGO se refleja equivalente a la variación de la cuenta Caja/Bancos siempre y cuando que no haya movimiento en los activos inmovilizados y recursos permanentes y distribución de dividendos.

\section{ANALISIS DE OTRAS FUENTES DE FONDOS NO CIRCULANTES}

¿Aumentó el capital social?, ¿se obtuvieron nuevos pasivos a largo plazo?, ¿se vendieron activos permanentes? Para responder estas inquietudes se deben examinar las cuentas que no son circulantes, en particular por el lado de los pasivos como recursos permanentes (Cuadro 12).
Cuadro 12. Variación de los recursos permanentes.

\begin{tabular}{lrrc}
\hline Concepto en \$ & 2002 & \multicolumn{1}{c}{2003} & Variación \\
\hline Pasivo a largo plazo & 70,000 & 90,000 & 20,000 \\
Capital social & 400,000 & 400,000 & \\
$\begin{array}{l}\text { Aumento (Reducción) de } \\
\text { Fuente de Fondos }\end{array}$ & & & 20,000 \\
\hline
\end{tabular}

\section{ANÁLISIS DE OTRAS APLICACIONES DE FONDOS NO CIRCULANTES}

¿Se adquirieron activos permanentes?, ¿se cancelaron pasivos a largo plazo? Para responder estas preguntas se deben examinar en particular las cuentas de inmovilizados (Cuadro 13). Vale anotar que en la cuenta activos fijos se debe considerar los valores brutos.

Cuadro 13. Variación de las cuentas inmovilizadas.

\begin{tabular}{lccc}
\hline Concepto en \$ & 2002 & 2003 & Variación \\
\hline $\begin{array}{l}\text { Inversiones permanentes } \\
\text { Edificaciones \& Planta }\end{array}$ & 50,000 & 50,000 & \\
$\begin{array}{l}\text { (Bruto) } \\
\text { Aumento (Reducción) }\end{array}$ & $1,000,000$ & $1,100,000$ & 100,000 \\
Aplicaciones de Fondos & & & \\
\hline
\end{tabular}

\section{INTERPRETACIÓN DEL FLUJO DE FONDOS}

Como se ha mencionado en la Parte I del trabajo, el flujo de fondos se puede presentar de una diversidad de formas, dependiendo del propósito deseado y de la problemática que uno desea abordar y resolver.

\section{Forma Alternativa: Flujo de Fondos como Recursos Totales.}

La forma convencional de presentación del cuadro de flujo de fondos a partir de las partidas del Balance, lo que corresponde al periodo 2003, se indica en el Cuadro 14.

Cuadro 14. Flujo de fondos como recursos totales en \$.

\begin{tabular}{lrlr}
\hline \multicolumn{1}{c}{ Fuente de Fondos } & \multicolumn{2}{c}{ Uso de Fondos } \\
\hline Utilidad del ejercicio & 50,000 & Caja y bancos & 10,000 \\
Depreciación & 50,000 & Cuentas por cobrar (clientes) & 30,000 \\
Cuentas por pagar (proveedores) & 30,000 & Existencias & 50,000 \\
Documentos por pagar & 30,000 & Gastos devengados por pagar & 10,000 \\
Sobregiros & 10,000 & Edificaciones \& Planta (Bruto) & 100,000 \\
Gastos pagados por adelantado & 10,000 & & \\
Total Fuentes & $\mathbf{2 0 0 , 0 0 0}$ & Total Usos & $\mathbf{2 0 0 , 0 0 0}$ \\
\hline
\end{tabular}


Cuadro 15. Flujo de fondos modificado como recursos totales en \$.

\begin{tabular}{lrlr}
\hline \multicolumn{1}{c}{ Fuente de Fondos } & \multicolumn{2}{c}{ Uso de Fondos } \\
\hline Fondos Generados por Operaciones (FGO) & 160,500 & Aumento Fondo de Maniobra & 10,000 \\
Pasivos a largo plazo & 20,000 & Edificaciones \& Planta & 100,000 \\
& & Dividendos & 60,500 \\
Total Fuentes & 180,500 & Total Usos & 180,500 \\
\hline
\end{tabular}

Un cuadro de flujo de fondos modificado que explica con mejor información una correcta interpretación financiera de cómo se ha financiado las inversiones y sus repercusiones en el largo plazo, se explicita en el Cuadro 15.

2. Forma Alternativa: Flujo de Fondos como Fondo de Maniobra

En el Cuadro 16 se examina y explica cómo a partir de los FGO, las fuentes y aplicaciones de cuentas no circulantes, se puede obtener la variación del Fondo de Maniobra. Se grafica con énfasis las decisiones financieras con repercusiones a largo plazo. Se aprecia que las operaciones comerciales (FGO) están sosteniendo con el $89 \%$ de la fuente de fondos y el crédito a largo plazo con el saldo, contribuyendo a la adquisición de activos fijos que usa el 56\% de los recursos, a la aplicación de dividendos (33\%) y al aumento del FM de manera estructural que representa el $11 \%$ de uso de los recursos generados.

Cuadro 16. Flujo de fondos como FM en \$

\begin{tabular}{lrr}
\hline Utilidad neta & 110,500 & \\
Más Depreciación y amortizaciones & 50,000 & \\
Fondos Generados por Operaciones (FGO) & 160,500 \\
Otras fuentes de fondos & 20,000 \\
$\begin{array}{l}\text { Aumento Pasivo a largo plazo } \\
\text { Aumento Capital social }\end{array}$ & 20,000 \\
TOTAL FUENTE DE FONDOS & 180,500 \\
TOTAL APLICACIÓN DE FONDOS & 160,500 \\
$\begin{array}{l}\text { Aumento Activos fijos } \\
\text { Dividendos repartidos }\end{array}$ & 100,000 \\
Aumento (Reducción) Fondo de Maniobra & 60,500 & \\
\hline
\end{tabular}

Como parte del análisis es viable vincular el cambio del FM con los cambios en la NOF (Cuadro 17), que comprende las cuentas del circulante (excepto la partida Caja/Bancos) que forman parte del ciclo de caja de la empresa, con el objeto de determinar su impacto en la variación de la partida Caja/Bancos.
Cuadro 17. Los cambios en la NOF en \$

\begin{tabular}{lrr}
\hline Aumento (Reducción del FM) & (1) & 20,000 \\
Variación en la Necesidad Operativa & & \\
de Fondos (NOF) & $(2)$ & $(10,000)$ \\
(menos) Aumento de clientes & $(30,000)$ & \\
(menos) Aumento de existencias & $(50,000)$ & \\
(más) Aumento de proveedores & 30,000 & \\
(más) Aumento Otros APC & 40,000 \\
Aumento (Reducción) CAJA/BANCOS & 20,000 \\
\multicolumn{2}{c}{$(3=1+2)$} \\
\hline
\end{tabular}

3. Forma Alternativa: Flujo de Fondos como Liquidez de la Empresa

En este esquema (Cuadro 18) se resalta en primer lugar la incidencia de los FGO en los cambios de las partidas vinculadas directamente con el ciclo de caja del negocio para obtener el nivel de caja generada por las operaciones (CGO) y de esta manera explicar y entender el impacto que causa en el nivel de liquidez de la empresa, al considerar la variación de los recursos permanentes e inmovilizados.

Cuadro 18. Flujo de fondos como liquidez de la empresa.

\begin{tabular}{lrr}
\hline Utilidad neta en \$ & 110,500 & \\
Más depreciación y amortizaciones & 50,000 & \\
FGO (1) & & 160,500 \\
Variación en la NOF (2) & $(10,000)$ \\
Menos Aumento Clientes & $(30,000)$ & \\
Menos Aumento Existencias & $(50,000)$ & \\
Más Aumento Proveedores & 30,000 & \\
Más Aumento Otros APC & 40,000 & \\
Caja Generada por Operaciones (CGO) & & 150,500 \\
(3=1+2) & & 20,000 \\
Otras Fuente de Caja No Circulantes (4) & 20,000 & \\
Aumento Pasivo largo plazo & & 170,500 \\
Aumento Capital social & & 160,500 \\
TOTAL FUENTES DE CAJA (5=3+4) & \\
APLICACIONES DE CAJA No Circulan- & & \\
tes (6) & 100,000 & \\
Aumento Activos fijos & 60,500 & \\
Dividendos entregados & & \\
Aumento (Reducción) CAJA/BANCOS & \\
(7=5-6) & & 10,000 \\
Caja inicial principios 2003 & & \\
Caja final finales 2003 & & 40,000 \\
\hline
\end{tabular}


Este es un caso atípico donde la NOF es negativa; lo común es tener la NOF positiva. De inmediato surge la necesidad de explorar ¿cuáles son las razones? Si se conoce que la necesidad de fondos aumenta con la actividad, o sea con el nivel de ventas, la pregunta inmediata sería ¿están disminuyendo las ventas? Un análisis más detallado implica examinar los niveles de ventas en periodos anteriores y la evolución de las políticas financieras. Aparentemente en la ilustración, la variación de las cuentas Otros APC se han transformado en fuentes de fondos que sumados a un mayor incremento de proveedores ha permitido cubrir en exceso las necesidades de fondos para cubrir las variaciones incrementales de clientes y existencias. Dicha situación ha producido que disminuya la caja disponible (CGO) como fuente de fondos en la contribución del incremento de la cuenta Caja/ Bancos.

\section{CONCLUSION}

El enfoque desarrollado en esta parte del trabajo le facilitará al lector abordar problemáticas similares y por tanto arribar a resultados relevantes que reforzarán y harán más eficaz su trabajo en la gestión financiera de los negocios.

\section{Notas}

Porlles, J. (2005), pp. 69.

Porlles, J. (2005). Op. cit., pp. 67-68.

Anthony, R. y Reece, J. (1982).

Porlles, J. (2005). Op. cit., pp. 65.

Porlles, J. (2005). Op. cit., pp. 65-66

Arbulú, J., y Stok, J. (2002), pp. 28 y 31

7 Ross, S.; Westerfield, R. y otros (2000), pp. 826-828.

8 Arbulú , J., y Stok, J. (2002). Op. cit., pp. 28-29.

9 Se hace la salvedad que para efecto de análisis se han separado los requerimientos de caja mínima

\section{BIBLIOGRAFIA}

Anthony, R. y Reece, J. (1982). Tercera edición. Principios Contables. Argentina, El Ateneo Editorial.

Arbulú, J., y Stok, J. (2002). El Modelo de la Necesidad Operativa de Fondos y el Capital de Trabajo. Revista de Egresados del PAD, Diciembre 2002. Escuela de Dirección de la Universidad de Piura.

Porlles, J. (2005). Flujo de Fondos: Poderosa Herramienta de Gestión en los Negocios (Parte I). En: Revista Gestión en el Tercer Milenio, Volumen 8 , N $N^{0} 15$, Julio 2005. Facultad de Ciencias Administrativas. UNMSM.

Ross, S.; Westerfield, R. y otros (2000) Quinta edición. Finanzas Corporativas. México, Editorial Mc Graw Hill. 
ANEXO 1: INDUSTRIAL TEXTIL S.A. - BALANCE GENERAL EN \$

\begin{tabular}{|c|c|c|c|}
\hline CONCEPTO & AL 31.12.02 & AL 31.12.03 & VARIACIÓN 03-02 \\
\hline \multicolumn{4}{|l|}{ Activos } \\
\hline \multicolumn{4}{|l|}{ Activos Circulantes } \\
\hline Caja y bancos & 30,000 & 40,000 & 10,000 \\
\hline Inversiones en valores & 10,000 & 10,000 & \\
\hline Cuentas por cobrar (clientes) & 170,000 & 200,000 & 30,000 \\
\hline Existencias & 130,000 & 180,000 & 50,000 \\
\hline Gastos pagados por adelantado & 30,000 & 20,000 & $(10,000)$ \\
\hline Total AC & 370000 & 450,000 & \\
\hline \multicolumn{4}{|l|}{ Activos Fijos } \\
\hline Edificaciones \&Planta & $1,000,000$ & $1,100,000$ & 100,000 \\
\hline - (Depreciación Acumulada) & 550,000 & 600,000 & 50,000 \\
\hline Edificaciones \&Planta (Neto) & 450,000 & 500,000 & \\
\hline Total Activos Fijos & 450,000 & 500,000 & \\
\hline Inversiones permanentes & 50,000 & 50,000 & \\
\hline Total Activos & 870,000 & $1,000,000$ & \\
\hline \multicolumn{4}{|l|}{ Pasivo y Patrimonio } \\
\hline \multicolumn{4}{|l|}{ Pasivo Circulante } \\
\hline Sobregiros & 20,000 & 30,000 & 10,000 \\
\hline Cuentas por pagar ( proveedores) & 50,000 & 80,000 & 30,000 \\
\hline Documentos por pagar & 40,000 & 70,000 & 30,000 \\
\hline Gastos devengados por pagar & 40,00 & 30,000 & $(10,000)$ \\
\hline Total PC & 150,000 & 210,000 & \\
\hline Pasivo a largo plazo (PLP) & 70,000 & 90,000 & 20,000 \\
\hline Total Pasivo & 220,000 & 300,000 & \\
\hline \multicolumn{4}{|l|}{ Patrimonio } \\
\hline Capital social & 400,000 & 400,000 & \\
\hline Utilidades retenidas & 250,000 & 300,000 & 50,000 \\
\hline Total Patrimonio & 650,000 & 700,000 & \\
\hline Total Pasivo y Patrimonio & 870,000 & $1,000,000$ & \\
\hline
\end{tabular}

ESTADO DE RESULTADOS 2003 EN \$

\begin{tabular}{lr}
\hline Ventas 2,000,000 & $2,000,000$ \\
\hline Costo de ventas & $1,550,000$ \\
Utilidad bruta & 450,000 \\
Gastos de venta y administración & 230,000 \\
Utilidad de operación & 220,000 \\
Gastos financieros & 20,000 \\
Utilidad antes de impuestos (UAI) & 200,000 \\
Impuesto a la renta & 89,500 \\
Utilidad después de impuestos ( U. Neta) & 110,500 \\
Dividendos & 60,500 \\
Utilidades retenidas & 50,000 \\
& \\
Anotaciones: & \\
Depreciación 2003 (incluido en el costo de venta) & 50,000 \\
Gastos de Explotación ( desembolsables) & \\
Gastos de venta y administración & 230,000 \\
Gastos financieros & 20,000 \\
Impuesto a la renta & 89,500 \\
Total gastos de explotación & 339,500 \\
\hline
\end{tabular}

